\title{
Nasal absorption of hydroxocobalamin in healthy elderly adults
}

\author{
D. Z. B. van Asselt, F. W. H. M. Merkus, F. G. M. Russel \& W. H. L. Hoefnagels \\ Department of Geriatric Medicine (DZBvA, WHLH), University Hospital Nijmegen, Centre of Bio-Pharmaceutical Sciences (FWHMM), \\ University of Leiden, and Department of Pharmacology (FGMR), Faculty of Medical Sciences, University of Nijmegen, The Netherlands
}

\begin{abstract}
Aims To investigate the nasal absorption of hydroxocobalamin in 10 healthy elderly adults.

Methods In a cross-over study, blood samples were collected before administration of the drug and after 10, 20, 30, 40, 60, 120, 180 and $240 \mathrm{~min}$. The plasma cobalamin concentration was determined by competitive radioisotope binding technique.

Results The maximal plasma cobalamin concentration $\left(C_{\max }\right)$ after nasal administration of $750 \mu \mathrm{g}$ hydroxocobalamin was $1900 \pm 900 \mathrm{pmol}^{-1}$ (mean \pm s.d.). The maximal plasma cobalamin concentration was reached in $35 \pm 13 \mathrm{~min}\left(t_{\max }\right)$. The $C_{\max }$ after nasal administration of $1500 \mu \mathrm{g}$ hydroxocobalamin was $3500 \pm 2500 \mathrm{pmol}^{-1}$ with a $t_{\max }$ of $28 \pm 16 \mathrm{~min}$. Both the $\operatorname{AUC}(0,240 \mathrm{~min})$ and AUC $(0,00)$ increased significantly with an increase of the dose from $750 \mu \mathrm{g}$ to $1500 \mu \mathrm{g}(P=0.037$ and $P=0.028$, respectively). The nasal spray was well tolerated. No signs of irritation or local sensitivity were noted.

Conclusions The nasal absorption of hydroxocobalamin in healthy elderly adults is rapid, high and well tolerated.
\end{abstract}

Keywords: hydroxocobalamin (vitamin B12) nasal spray, nasal absorption, elderly volunteers, plasma cobalamin concentrations

\section{Introduction}

Cobalamin (vitamin B12) deficiency is especially common among elderly people. Studies indicate that as many as $15 \%$ are cobalamin deficient $[1,2]$. The treatment of choice is the administration of cobalamin by intramuscular injections $[3,4]$. Usually, the patient is put on a lifetime regimen of monthly maintenance injections. Intramuscular injections, however, have disadvantages: they are inconvenient, painful and the frequent need for the assistance of health professionals makes the patient dependent and increases the cost of treatment considerably. These drawbacks could encourage noncompliance. A more convenient and cost-effective alternative would be advantageous to health care in general and patients in particular.

The search for alternative routes of cobalamin administration began soon after cyanocobalamin was isolated and introduced for parenteral use in 1948 [5]. Nasal inhalation, insufflation or instillation of cyanocobalamin were proposed in the early 1950s [6-8]. The formulations for nasal inhalation or instillation consisted of low concentrations of cyanocobalamin in isotonic saline solution or lactose powder. Although these formulations were reported to be effective in the treatment of pernicious anaemia, none of these proposals found a follow-up in clinical practice, in industry or in the scientific literature. Recently a gel for nasal

Correspondence: Mrs Dieneke van Asselt, MD, Department of Geriatric Medicine, University Hospital Nijmegen, PO Box 910I, 6500 HB Nijmegen, The Netherlands. cyanocobalamin instillation has become commercially available. The gel, in a dose of 400-500 $\mu \mathrm{g}$ cyanocobalamin, has been claimed to be safe and effective [9]. We are not aware of studies that show the safety and efficacy of the nasal gel in treating cobalamin deficiency. A practical problem with the use of a gel is that it sometimes dries up and sticks to the unit-dose tubes. In the sixties oral cyanocobalamin administration for the treatment of pernicious anaemia was proposed [10-12]. Despite the claim that doses of $150 \mu \mathrm{g}$ to $1000 \mu \mathrm{g}$ daily are effective, it is rarely used [13].

A disadvantage of all these forms of treatment is that they contain cyanocobalamin while hydroxocobalamin is the drug of choice. Since the fifth World Health Organization Model List of Essential Drugs, hydroxocobalamin, and not cyanocobalamin, has been the cobalamin included in the list [14]. Hydroxocobalamin binds more extensively to plasma proteins and has a longer half-life in the body than cyanocobalamin. As a result, hydroxocobalamin is better retained in the body and therefore requires less frequent dosage [15]. Moreover, cyanocobalamin is contraindicated in patients with tobacco and tropical amblyopia and optic neuropathy in pernicious anaemia. A plea for the withdrawal of cyanocobalamin has been made $[16,17]$.

Recently, a formulation for the nasal administration of hydroxocobalamin has been developed [18]. Here we present the results of our exploratory study on the absorption of nasally administered hydroxocobalamin in healthy elderly adults. To our knowledge this is the first report to document nasal hydroxocobalamin absorption in humans. 


\section{Methods}

Subjects

Healthy elderly adults were recruited from the general population by means of advertisements in local newspapers. The following exclusion criteria were applied: use of vitamin supplements containing cobalamin, use of nasal medication, or acute or chronic rhinitis. Ten subjects were selected, six females and four males, age $71.6 \pm 4.6$ year (mean \pm s.d.).

\section{Protocol}

The study protocol was approved by the Committee for Experimental Research with Humans of the University Hospital Nijmegen. All subjects gave written informed consent. On the first test day subjects were randomly allocated to a dose of $750 \mu \mathrm{g}$ or $1500 \mu \mathrm{g}$ hydroxocobalamin. On the second test day (after $163 \pm 10$ days, range 147-175 days) subjects received the alternate dose. Blood samples were obtained through an indwelling cannula inserted into a forearm vein of each subject. After a blood sample was collected the cannula was flushed with $1 \mathrm{ml}$ of heparinized salt solution (150 IE heparin in $0.9 \% \mathrm{NaCl}$ solution) in order to maintain patency. Before each blood sample was collected the first 1-2 $\mathrm{ml}$ blood were discarded. EDTAplasma was collected before and after drug administration at the following time points: $0,10,20,30,40,60,120,180$ and $240 \mathrm{~min}$.

The hydroxocobalamin formulation contained $750 \mu \mathrm{g}$ hydroxocobalamin per $70 \mu \mathrm{l}$ in a preserved solution and the nasal spray device was a metered pump (Valois, France, VP 7/70). The investigator administered $750 \mu \mathrm{g}$ (one puff) or $1500 \mu \mathrm{g}$ (one puff in each nostril) hydroxocobalamin nasally. The nasal dispenser was weighed before and after administration of the drug to ensure that the dose had actually been given.

The plasma cobalamin concentration was determined by competitive radioisotope binding technique using purified hog intrinsic factor as cobalamin binder (Solid Phase DualCount, Diagnostic Products Corporation, Los Angeles, California). The within-assay coëfficient of variation was $3.1-3.5 \%$ and the between-assay coëfficient of variation was $3.3-6.7 \%$. The assay has a detection limit of $37 \mathrm{pmol}^{-1}$. In case the plasma cobalamin concentration fell outside the range of the assay, i.e. $>1700 \mathrm{pmol}^{-1}$, the samples were diluted with zero-diluent and assayed again.

\section{Pharmacokinetic analysis}

Pharmacokinetic parameters of cobalamin were analyzed by model-dependent and model-independent methods. Because a semi-logarithmic plot of the concentration-time data suggested a one-compartment model with first-order absorption, data were analyzed according to this model by weighted (1/y) nonlinear regression with use of PCNONLIN (version 3.0, Scientific Consulting Inc., Apex, N.C.). The following first-order absorption equation, which takes the endogenous concentration of cobalamin into account, was applied:

$$
\begin{gathered}
C(t)=\mathrm{A} \cdot\left(\mathrm{e}^{-k_{e l} \cdot t}-\mathrm{e}^{-k_{a} \cdot t}\right)+C \mathrm{~b} \\
\mathrm{~A}=\frac{F \cdot D \cdot \mathrm{k}_{\mathrm{a}}}{V\left(k_{\mathrm{a}}-k_{\mathrm{el}}\right)}
\end{gathered}
$$

in which $\mathrm{A}$ is the intercept of the elimination phase on the y-axis, $k_{\mathrm{el}}$ and $k_{\mathrm{a}}$ are the rate constants of elimination and absorption, $V$ is the volume of distribution, $D$ is the dose, $F$ is the bioavailability and $\mathrm{Cb}$ is the baseline plasma cobalamin concentration. The following pharmacokinetic parameters were calculated: total area under the curve, $\operatorname{AUC}(0, \infty)=\mathrm{A}\left(1 / k_{\mathrm{el}}-1 / k_{\mathrm{a}}\right) ;$ total area under the first moment curve, $\mathrm{AUMC}(0 \infty)=\mathrm{A}\left(1 / k_{\mathrm{el}}^{2}-1 / i t k_{\mathrm{a}}^{2}\right)$; apparent total plasma clearance, $\mathrm{CL} / F=D / \mathrm{AUC}$; apparent volume of distribution, $V / F=D \cdot A U M C /(A \cup C)^{2}-D /\left(A \cup C . k_{\mathrm{a}}\right)$; absorption half-life, $t_{1 / 2, \mathrm{a}}=\ln 2 / k_{\mathrm{a}}$; elimination half-life, $t_{1 / 2, \mathrm{z}}=\ln 2 / k_{\mathrm{el}}$; mean residence time, MRT $=\mathrm{AUMC} / \mathrm{AUC}$; mean absorption time, $\mathrm{MAT}=\mathrm{MRT}-1 / k_{\mathrm{el}}$; time to reach peak plasma concentration, $t_{\max }=\ln \left(k_{\mathrm{a}} / k_{\mathrm{el}}\right) /\left(k_{\mathrm{a}}-k_{\mathrm{el}}\right)$; and the peak plasma concentration, $C_{\max }$, is calculated after substitution of the value for $t_{\max }$ in the first order absorption equation.

The goodness of fit to the plasma concentration data was evaluated through the deviations between observations and model-predicted values, expressed as

$$
r^{2}=1-\Sigma(\mathrm{Dev})^{2} / \Sigma(\mathrm{Obs})^{2}
$$

AUC and AUMC were also calculated modelindependently by application of the linear trapezium method (from 0 to $240 \mathrm{~min}$ ) after correction for baseline plasma cobalamin concentrations, with extrapolation to infinity by using the log-linear terminal slope of the plot.

\section{Statistical analysis}

The results are presented as means \pm s.d. The Wilcoxon Signed Rank test was used for comparing continuous variables of paired samples. A $P$ value of 0.05 or less was considered statistically significant.

\section{Results}

Figure 1 shows the mean plasma concentrations of cobalamin after nasal administration of $750 \mu \mathrm{g}$ and $1500 \mu \mathrm{g}$ to 10 healthy elderly adults. The pharmacokinetic parameters are summarized in Table 1. Model-independent analysis revealed virtually the same values (data not shown). The goodness of fit expressed as the mean coefficient of determination was $0.98 \pm 0.14$ (range 0.939-0.998), indicating that the model described the data accurately. We assumed that the descending part of the plasma curves represents the elimination phase of hydroxocobalamin, because the rate constants $k_{\mathrm{el}}$ and $k_{\mathrm{a}}$ in the model are not uniquely identifiable. The only way to be sure of this assumption is to compare the calculated values with the elimination rate constant after intravenous administration. Literature data of intravenously administered hydroxocobalamin in dosages yielding plasma concentrations comparable to our data are not available. Although the model fitted the data very well it should be noticed that the extrapolated part of the AUC contributes 


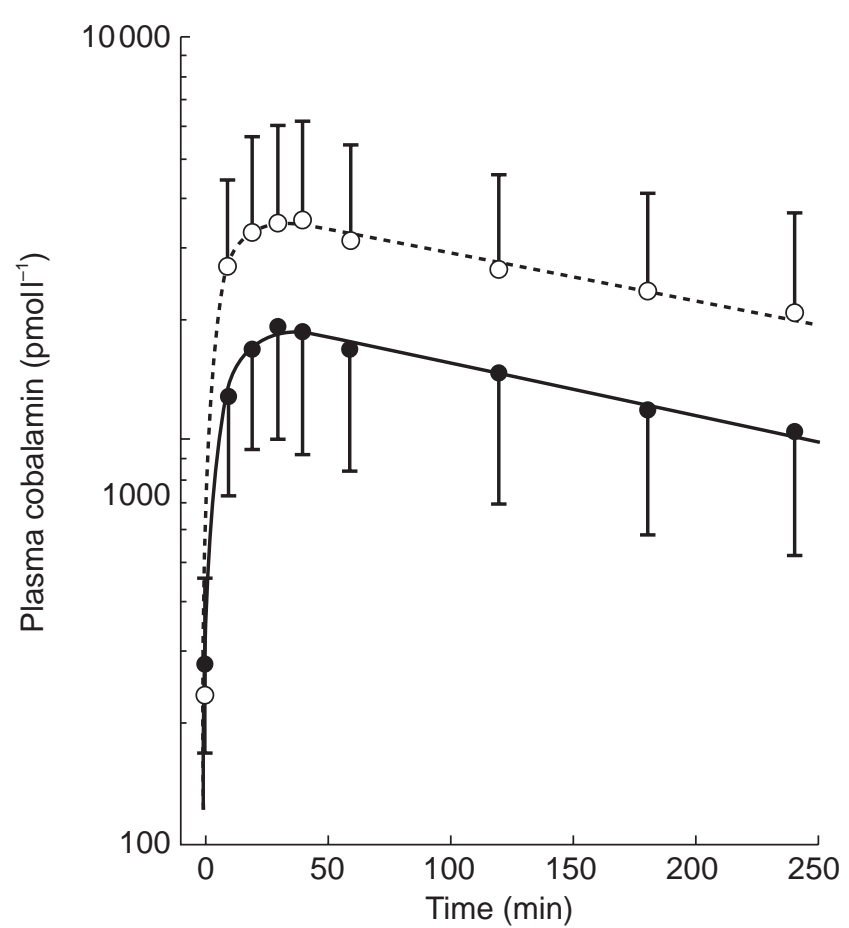

Figure 1 Mean and s.d. plasma cobalamin concentration after nasal administration of $750 \mu \mathrm{g}(\mathbf{O})$ and $1500 \mu \mathrm{g}(\bigcirc)$ hydroxocobalamin in healthy elderly adults.

to a large extent to the total AUC (44-55\%). Extrapolation of the terminal phase of the plot to infinity may be an underestimation of the actual AUC, because an additional, slower phase, might have been missed. However, since plasma concentrations of all individuals at both dosages had nearly returned to their baseline values after $48 \mathrm{~h}$ (data not shown), the influence of such an underestimation on the total AUC would be relatively minor.

The nasal absorption of hydroxocobalamin was fast. The $C_{\max }$ after nasal administration of $1500 \mu \mathrm{g}$ hydroxocobalamin was approximately twice as high as the $C_{\max }$ after nasal administration of $750 \mu \mathrm{g}$ hydroxocobalamin. However, due to intra- and intersubject variability the difference in $C_{\max }$ was not statistically significant $(P=0.09)$. Nevertheless, both the $\operatorname{AUC}(0,240 \mathrm{~min})$ and $\operatorname{AUC}(0, \infty)$ increased significantly with the increase in dose $(P=0.04$ and $P=0.03$, respectively).

The nasal spray was well tolerated. No signs of irritation or local sensitivity were noted.

\section{Discussion}

The results of our study show that nasal hydroxocobalamin absorption is rapid, high, and safe. The absorption of watersoluble compounds depends on several factors like the contact time with the nasal mucosa and the size of the compound. There is an inverse relationship between the molecular weight of a drug and the absorbed proportion of the intranasal dose [19]. Nasal absorption decreases with increasing molecular weight but the absorption of compounds up to about 1000 daltons is good [20]. Nevertheless, this study demonstrates that the absorption of hydroxocobalamin with a molecular weight of 1346 daltons is fair. Other hydrophilic compounds of similar weight like oxytocin $(\mathrm{MW}=1007), \quad$ desmopressin $\quad(\mathrm{MW}=1069), \quad$ buserelin $(\mathrm{MW}=1238)$, and nafarelin $(\mathrm{MW}=1321)$ are also absorbed nasally. These products have been on the market for some years. The nasal absorption of relatively large hydrophilic compounds is thought to occur by diffusion through aqueous channels in the epithelial membrane [19]. The role of the cobalamin-binder haptocorrin, which has been demonstrated in nasal secretion, [21] in the nasal absorption of hydroxocobalamin is unclear.

Our study presents the first results on the nasal absorption of hydroxocobalamin in healthy elderly volunteers. We found that nasally administered hydroxocobalamin is rapidly absorbed, that the amount absorbed increases significantly with increasing dose and that it is well tolerated. The plasma concentrations achieved after nasally administered hydroxocobalamin are in the therapeutic range. We did not compare the intranasal administration with the currently used intramuscular route. However, based on values in literature it can be estimated that the bioavaliabilty of nasal hydroxocobalamin is approximately $2-5 \%$. Plasma concen-
Table 1 Pharmacokinetic parameters of nasal hydroxocobalamin.

\begin{tabular}{|c|c|c|c|}
\hline \multirow[b]{2}{*}{ Parameter } & \multicolumn{3}{|c|}{ Nasal hydroxocobalamin } \\
\hline & $750 \mu g$ & $1500 \mu g$ & P-value \\
\hline$C_{\max }\left(\operatorname{pmol}^{-1}\right)$ & $1900 \pm 900$ & $3500 \pm 2500$ & 0.09 \\
\hline$t_{\max }(\min )$ & $35 \pm 13$ & $28 \pm 16$ & 0.17 \\
\hline $\mathrm{Cb}\left(\mathrm{pmoll}^{-1}\right)$ & $280 \pm 170$ & $240 \pm 70$ & 1.00 \\
\hline $\operatorname{AUC}(0,240 \mathrm{~min})\left(\mathrm{pmol} \mathrm{ml} \mathrm{min}{ }^{-1}\right.$ & $270 \pm 160$ & $580 \pm 460$ & 0.04 \\
\hline $\operatorname{AUC}(0, \infty)\left(\mathrm{pmol} \mathrm{ml} \mathrm{min}^{-1}\right)$ & $480 \pm 350$ & $1220 \pm 1130$ & 0.03 \\
\hline$t_{1 / 2, \mathrm{a}}(\min )$ & $7.6 \pm 3.3$ & $5.3 \pm 3.6$ & 0.15 \\
\hline$t_{1 / 2, \mathrm{z}}(\min )$ & $170 \pm 60$ & $200 \pm 70$ & 0.06 \\
\hline MRT (min) & $260 \pm 80$ & $300 \pm 110$ & 0.08 \\
\hline MAT (min) & $11 \pm 5$ & $8 \pm 5$ & 0.15 \\
\hline $\mathrm{CL} / F\left(\mathrm{ml} \mathrm{min}^{-1}\right)$ & $2.4 \pm 2.3$ & $2.1 \pm 1.8$ & 0.72 \\
\hline$V / F(1)$ & $0.52 \pm 0.49$ & $0.50 \pm 0.33$ & 0.88 \\
\hline
\end{tabular}

Data are means \pm s.d. $\mathrm{AUC}(0,240 \mathrm{~min})$ was calculated by applying the linear trapezoidal rule. $C_{\max }$, peak plasma concentration; $\mathrm{Cb}$, endogenous plasma cobalamin concentration; $t_{\max }$, time to reach the peak plasma concentration; AUC, area under the plasma concentration-time curve; $t_{1 / 2, a}$, absorption half-life; $t_{1 / 2, z}$, elimination half-life; MRT, mean residence time; MAT, mean absorption time; CL/F, apparent total plasma clearance; $V / F$, apparent volume of distribution. 
trations achieved after intramuscular injections of comparable dosages are approximately 10 times higher than after nasal administration [22, 23]. However, with an intramuscular injection of $1000 \mu \mathrm{g}$ about $50 \%$ of free hydroxocobalamin is lost into urine [24]. Another, although less frequently applied route is oral administration. Clinical and haematological remission has been reported in patients with pernicious anaemia treated with $150 \mu \mathrm{g} \mathrm{day}^{-1}, 300 \mu \mathrm{g} \mathrm{day}^{-1}$ or $500 \mu \mathrm{g} \mathrm{day}^{-1}$ of oral hydroxocobalamin [10-12]. The achieved plasma concentrations after oral administration of hydroxocobalamin are about 10 to 20 times lower than after nasal administration of $750 \mu \mathrm{g}$ and $1500 \mu \mathrm{g}$, respectively [10-12].

The rather large intra- and intersubject differences in the amount of nasally absorbed hydroxocobalamin can be explained by differences in mucociliary clearance or variations in spraying technique and deposition pattern. These variables will have to be investigated in more detail in a next study. The nasal absorption of hydroxocobalamin is so high that weekly application could prove a good alternative for monthly injections. In a pilot study during 1 month of four patients with Crohn's disease the mean serum cobalamin concentration increased significantly after weekly administration of $1500 \mu \mathrm{g}$ of the same formulation [25]. A large clinical study will have to demonstrate that weekly nasal administration of hydroxocobalamin to cobalamin deficient patients results in normalization of the plasma cobalamin level and reversal of clinical consequences of cobalamin deficiency.

This study was supported by a grant of NESTOR, funded by the Ministry of Health, Welfare and Sports and the Ministry of Education, Culture and Sciences. The authors are indebted to the staff and personnel of the Laboratory of Endocrinology and Reproduction of the University Hospital Nijmegen for performing the cobalamin assay.

\section{References}

1 Lindenbaum J, Rosenberg IH, Wilson PWF, Stabler SP, Allen RH. Prevalence of cobalamin deficiency in the Framingham elderly population. Am J Clin Nutr 1994; 60: 2-11.

2 Pennypacker LC, Allen RH, Kelly JP, et al. High prevalence of cobalamin deficiency in elderly outpatients. J Am Geriatr Soc 1992; 40: 1197-1204.

3 Babior BM, Bunn HF. Megaloblastic anemia. In Harrison's principles of internal medicine, 12. ed., Wilson JD, Braunwald E, Isselbacher KJ, eds, New York: McGraw-Hill, Inc., 1991: 1523-1529.

4 Freedman ML. Blood disorders and their management in old age. In Textbook of geriatric medicine and gerontology, 4. ed., Brocklehurst JC, Tallis RC, Fillit HM, eds, Edinburgh: Churchill Livingstone, 1992: 859-922.

5 West R. Activity of vitamin B12 in Addisonian pernicious anemia. Science NY 1948; 107: 398.
6 Israëls MCG, Shubert S. The treatment of pernicious anemia by insufflation of vitamin B12. Lancet 1954; i: 341-343.

7 Monto RW, Rebuck JW, Brennan MJ. Crystalline B12 inhalation therapy in pernicious anemia. Am J Med Sci 1953; 225: 113-119.

8 Monto RW, Rebuck JW. Nasal instillation and inhalation of crystalline vitamin B12 in pernicious anemia. Arch Intern Med 1954; 93: 219-230.

9 Romeo VD, Sileno A, Wenig DN. Intranasal cyanocobalamin. JAMA 1992; 268: 1268-1269.

10 Waife SO, Jansen CJ, Crabtree RE, Grinnan EL, Fouts PJ. Oral vitamin B12 without intrinsic factor in the treatment of pernicious anemia. Ann Intern Med 1963; 58: 810-817.

11 Brody EA, Estren S, Wasserman LR. Treatment of pernicious anemia by oral administration of vitamin B12 without added intrinsic factor. New Engl J Med 1959; 260: 361-367.

12 Waife SO, Jansen CJ, Crabtree RE, Grinnan EL, Fouts PJ. Oral vitamin B12 without intrinsic factor in the treatment of pernicious anemia. Ann Int Med 1963; 58: 810-817.

13 Lederle FA. Oral cyanocobalamin for pernicious anemia: medicine's best kept secret? JAMA 1991; 265: 94-95.

14 Reidenberg MM. Cyanocobalamin - a case for withdrawal. J R Soc Med 1993; 86: 309.

15 Hall CA, Begley JA, Green Colligan PD. The availability of therapeutic hydroxocobalamin to cells. Blood 1984; 63: 335-341.

16 Freeman AG. Cyanocobalamin-a case for withdrawal. $J R$ Soc Med 1992; 85: 686-687.

17 Linnell JC, Matthews DM, England JM. Therapeutic misuse of cyanocobalamin. Lancet 1978; ii: 1053-1054.

18 Merkus FWHM. A pharmaceutical composition for the intranasal administration of hydroxocobalamin. International Patent Appl. WO 95/17164. 1995: 1-10.

19 Fisher AN, Brown K, Davis SS, Parr GD, Smith DA. The effect of molecular size on the nasal absorption of watersoluble compounds in the albino rat. J Pharm Pharmacol 1987; 39: $357-362$.

20 McMartin C, Hutchinson LE, Hyde R, Peters GE. Analysis of structural requirements for the absorption of drugs and macromolecules from the nasal cavity. J Pharm Sci 1987; 76: 535-540.

21 Tonnesen P, Thim L, Nexo E. Epidermal growth factor and haptocorrin in nasal secretion. Scand J Clin Lab Invest 1990; 50: 187-194.

22 Skouby AP. Retention and distribution of B12 acitivity and requirement for B12 following parenteral administration of hydroxocobalamin (Vibiden). Acta Med Scand 1966; 180: 95-105.

23 Hertz H, Ostergaard Kristensen HP, Hoff-Jorgensen E. Studies on vitamin B12 retention. Scand J Haematol 1964; 1: $1-15$.

24 Killander A, Schilling RF. Studies on hydroxocobalamin. J Lab Clin Med 1961; 57: 553-561.

25 Bruins Slot W, Merkus FWHM, Tytgat GNJ. Hydroxocobalamin intranasally in patients with Crohn's disease and low serum B12 levels. Gastroenterology 1996; 110: A874.

(Received 6 May 1997, accepted 2 September 1997) 